\title{
Image Clustering Method based on Particle Swarm Optimization
}

\author{
Iuliia Kim, Anastasiia Matveeva and Ilya Viksnin \\ St. Petersburg National Research University of Information Technolo- \\ gies, Mechanics and Optics \\ Kronverkskiy Prospekt, 49, Saint-Petersburg, 197101, Russian Federa- \\ tion \\ Email: yulia1344@gmail.com, anastasiamatveevaitmo@gmail.com, \\ wixnin@cit.ifmo.ru
}

\author{
Igor Kotenko \\ St. Petersburg Institute for Informatics \\ and Automation of the Russian Acad- \\ emy of Sciences (SPIIRAS) \\ 14-th Liniya, 39, Saint-Petersburg, \\ 199178, Russia \\ Email: ivkote@comsec.spb.ru
}

\begin{abstract}
To implement efficient computer vision mechanisms, efficient image clustering methods are important. The paper elaborates a clustering method based on particle swarm optimization (PSO) which provides automatic establishment of clustering parameters. The developed PSO based clustering method was tested on 860 images for a car vision system and its results and contribution to the pattern recognition quality improvement were assessed in comparison with fuzzy $\mathrm{C}$-means and $k$-means. The results do not differ significantly, but distinction in average time of work for these methods was noted. The PSO clustering method is faster than $k$-means and slower than fuzzy $C$-means. However, fuzzy $C$-means method does not guarantee correct results during the further analysis, so the PSO clustering method can be more efficient for implementation in computer vision systems.
\end{abstract}

\section{INTRODUCTION}

$\mathrm{C}$ OMPUTER vision is one of the most prospective fields in different spheres of life [1-4]. It is a wide area of theoretical materials and technical methods connected with object detection, object tracking and object classification. It can be embedded in devices in order to automate their working processes and make them perform pattern recognition tasks.

To provide the correct work of pattern recognition, it is necessary to elaborate efficient methods of image processing; and clustering is one of them. However, many existing clustering methods depend on manual tuning and do not guarantee accurate results in case of visual information distortion.

Usage of machine vision is one of the most important challenge of automatization in different spheres of social life. Authors study implementation of machine vision for autonomous vehicles. Main tasks of such research are defining of other road users (autonomous cars), recognition of road infrastructure and road marking [5-7].

Most of the existing solutions are particular - it seems necessary to make some adjustments to use such solutions in other conditions. Some of the approaches for organizing machine vision need a lot of computing power.

The paper is dedicated to the ways of pattern recognition quality improvement. Relevance of the proposed paper to the computer vision area can be explained by the fact that computer vision can be embedded in cars, drones and used in order to automate such process as road traffic and space monitoring. It is vital to provide a correct work of computer vision, because its errors can have fatal consequences: victims and damages.

In order to avoid such consequences, the authors state the purpose to elaborate efficient methods of image processing. Among them it is possible to call segmentation and clustering. However, they do not guarantee accurate results in case of visual information distortion or high noise level. The reason is in the need for manual tuning of these methods.

The authors of the paper tried to develop a clustering method based on the particle swarm optimization (PSO) that sets parameters automatically. This method also includes $k$-means clustering method, which is intended to group pixels into predetermined number of clusters by calculating the minimum value of distance function. Relative to the PSO, the particle group motion (pixel-by-pixel passage through the image) and search for the best solution for the entire swarm (search for pixel with a maximum average intensity value in a certain region) are used.

In comparison with the original $k$-means method, the need of cluster amount predetermining by user is eliminated, and apart from the operation of distance function minimization the operation of color function minimization is added. An object in an image (or a part of an object) is characterized with relative color uniformity, i.e. if the distance function for pixels $a$ and $b$ that belong to one object tends to minimum, their color function also tends to minimum. In this paper experiments with non-spoiled images are represented in order to prove preparedness of this method for implementing in process of work with distorted visual information.

Further structure of this paper is as follows. Section 2 provides the overview of the relevant works and sets the theoretical background. Section 3 considers the research task statement and the suggested clustering method based on particle swarm optimization. Section 4 demonstrates the experimental results. Conclusions and future research directions are outlined in section 5 . 


\section{RELATED WORK AND THEORETICAL BACKGROUND}

One of the main purposes in the area of computer vision is the error percentage reduction in the pattern recognition. In this paper the pattern recognition error is a situation when the needed object in the image either is not detected or is detected incorrectly. The pattern recognition process consists in three basic stages:

- filtering and image preparation for the analysis; the image preparation includes image compression, selection of needed regions, ridding of noise;

- logical processing of filtering results, which is responsible for object detection in the filtered image;

- decision-making based on the results of the logical processing, which implies classification of the detected objects.

Image segmentation [8] is related to the ways of increasing the accuracy of recognition. It is a process of dividing a digital image into a set of its constituent regions in order to select objects and their boundaries. As there is no general solution for the image segmentation task, different methods and algorithms were created, which are oriented to the determined categories of images. Depending on the category of the image, its priority properties and then the ways of grouping them are chosen.

The methods listed below can serve as examples:

- active contour method [9] (deformation of original image contours to the boundaries of the specified objects);

- topological alignment method (matching two consecutive frames in the image stream);

- watershed method [10] (establishment of boundary watersheds between different segments according to the determined rule).

As for semantic part [11-13] of the visual information, the following kinds of image segmentation can be distinguished:

- semantic segmentation based on the use of Fully Convolutional Networks (pixel-to-pixel mapping without prior allocation of specific areas);

- weakly supervised semantic segmentation (with use of bounding frames and special labels on the images);

- region-based semantic segmentation (region allocation based on predetermined grouping rules).

One of the segmentation methods is clustering. Image clustering is a division of pixels into several non-intersecting groups (clusters) in such a way that pixels from the same group have similar features, meanwhile the features of pixels from different groups vary significantly from each other.

Clustering task statement:

1) There are: $X$ - a set, which consists of $N$ objects; $C$ a set, which consists of $M$ identifiers, such as number, name or label; the distance function between objects $d\left(x, x^{\prime}\right)$, where $x$ and $x^{\prime}$ are two objects in the image. The distance function is represented in (1):

$$
d\left(x ; x^{\prime}\right)=\sqrt{\left(x-x^{\prime}\right)^{2}}
$$

2) It is necessary to divide the set of objects $X$ into $M$ non-intersecting subsets (clusters) in such a way that each cluster was represented as an aggregate of objects from the set $X$, whose distance function values $d$ are closed to each other. In addition, the following conditions must be fulfilled:

- each cluster is assigned a cluster identifier $C_{j} ; j \in$ $[1 ; M]$ (number, name, label);

- each object $x_{i} ; i \in[1 ; N]$ can belong to one and only one cluster.

There are plenty of different clustering methods. Some of the most spread examples of these methods are $k$-means and fuzzy $C$-means. The idea of the $k$-means algorithm is to minimize the distance between objects in the clusters. The algorithm stops working when the further minimization becomes impossible.

The main step of the $k$-means algorithm:

1) At the beginning of the algorithm the quantity of clusters is set and then, according to the determined rule, centroids are allocated (centers of mass of clusters). The minimizing function is represented in (2):

$$
J=\sum_{i=1}^{N} \sum_{k=1}^{M} d\left(x_{i}, c_{k}\right)
$$

where $X-\mathrm{a}$ set of clustering objects, $x_{i} \in X$ a clustering object, $i \in[1 ; N], C-$ a set of clusters, $c_{k} \in$ $C$ - centroid, $k \in[1 ; M], M$ - an amount of clusters, $N$ - an amount of objects, $d-$ a value of distance function between object and centroid.

2) Each object correlates with the determined cluster by calculating the value of the distance function between this object and each center of mass and then selecting the least one among the calculated values. After that the centers of mass of clusters are recalculated, as in (3):

$$
c_{j}=\frac{\left(\sum_{t=1}^{T}\left(x_{t}\right)\right)}{T}
$$

where $x_{t} \in C_{j} ; t \in[1 ; T] ; T-$ an amount of objects in the cluster $C_{j} ; j-$ a cluster number, $j \in[1 ; M] ; M-$ an amount of clusters.

3) If $c_{j}=c_{j}-1$, it means that object clustering is completed, otherwise it is necessary to return to the second step and recalculate centroids again.

Fuzzy $C$-means algorithm is based on the fuzzy logic, i.e. on the assumptions that each clustering object from the set $X$, which consists of $N$ objects to some extent belongs to a particular cluster from the set of clusters $C$.

The main step of the fuzzy $C$-means algorithm:

1) As input values, there are: $M$ - an amount of clusters, $1<m<\infty-$ a measure of accuracy, $0<\varepsilon<1-$ a criterion of the end, $U_{0}=u_{i j}\left(x_{i} ; c_{j}\right): x_{i} \in X ; c_{j} \in C-$ 
a weighting matrix of belonging of the clustering object $x_{i} \in X ; i \in[1 ; N]$; to the cluster $C_{j} \in C ; j \in[1$; $M] ; 0<u_{i j}<1$ :

The minimizing function is shown in (4):

$$
J=\sum_{i=1}^{N} \sum_{k=1}^{M} u_{i j}^{m} \cdot d\left(x_{i}, c_{k}\right)
$$

2) Then the centroids are calculated, as in (5):

$$
c_{j}=\frac{\left(\sum_{i=1}^{N} u_{i j}^{m} \cdot x_{i}\right)}{\sum_{i=1}^{N} u_{i j}^{m}}
$$

3) After this the weights are recalculated, as in (6):

$$
u_{i j}=\frac{1}{\sum_{k=1}^{M}\left(\frac{d\left(c_{j}, x_{i}\right)}{d\left(c_{k}, x_{i}\right)}\right)^{\frac{2}{m-1}}}
$$

4) The next step is to compare $\left|U_{k}-U_{k-1}\right|$ with the value of $\varepsilon$. In case the first value is less than the second one, the algorithm is finished, otherwise it is necessary to return to the step 2 and recalculate the centroids.

\section{Clustering Method BASED ON PARTICLE SWARM OPTIMIZATION}

The algorithms mentioned above have quite substantial drawbacks, such as:

- sensitivity to outliers (values that are allocated from the total sample);

- need for the user to specify the amount of clusters beforehand;

- presence of some uncertainty degree in the threshold parameter definition;

- uncertainty of actions with objects that possess the properties of two different clusters simultaneously;

- need for the user to specify clustering parameters.

Due to shortcomings of clustering algorithms represented by authors, there is such a situation when the clustering task can be solved incorrectly, namely: for the clustering objects $x_{i} \in X ; i \in[1 ; N]$ and the clusters $C_{j} \in C ; j \in[1 ; M]$ with the centroids $c_{j} \exists t \neq j g_{x i} \neq g_{c t} ; g_{x i}=g_{c j} ; x_{i} \in C_{t} ; x_{i} \notin C_{j}$; where $g_{x i}$ - a label of belonging of the $i^{t h}$ object to the cluster, $g_{c j}-$ a label of belonging of the centroid to the $j^{\text {th }}$ cluster.

In this way, authors of this paper have the following research task: it is necessary to find such centroids $c_{j}$ for the $C_{j} \in C$ that $\forall x_{i} \in X \exists ! C_{j} g_{x i}=g_{c j} ; x_{i} \in C_{j} ; g_{x i} \notin \mathrm{g}_{\mathrm{ca}} ; x_{i} \notin C_{a}$; $g_{x i} \neq g_{c b} ; x_{i} \notin \mathrm{C}_{\mathrm{b}} ; a \in[1 ; j-1] ; b \in[j+1 ; M]$.

To solve this pattern recognition quality improvement task, it is essential to exclude the user's direct participation in the process of specifying the amount of clusters and rule (or set of rules) during the initial centroid allocation.
Methods providing automated image segmentation and clustering were proposed in many scientific works. In the [14] processing of image segments for fruit peel defects detection was proposed. The source image is divided into segments, which are given as an input to Adaptive Artificial Neural Network (AANN). The image processing is automated, because AANN adapts to each input and its features.

Segmentation based on color maps was developed in the article [15]. Its authors in the research rely on four-color labeling theorem. They improved the existed four-color labeling method, which used random initial distribution and developed heuristic four-color labeling. This iterative algorithm generates more reasonable color maps with a global view of the whole image and provides better results in case of images with clutters and complicated structures.

In the paper [16] image clustering is used in order to provide automated retinal screening. In the described method heuristic based clustering is included. Initial centers are allocated according to measures defining statistical distribution of data is incorporated in the proposed methodology.

Another effective clustering method was proposed for heterogeneous disease expression data [17]. Recursive Kmeans spectral clustering method (ReKS) was developed, which was found to be superior to the hierarchical clustering method and much faster than $k$-means.

In the work [18] a novel data clustering algorithm was elaborated. It is based on heuristic rules, which are built according to $k$-nearest chain, and give an opportunity to get rid of the need in specifying the number of clusters. KNearest Neighbors Chain (KNNC) serves as basis for proposing two heuristic rules to find initial clusters and their proper amount. The first heuristic rule reflects the degree of separation of clusters and the second rule determines the inner compactness of a cluster.

In order to resolve the issue of arbitrary choices on clustering parameters, authors decided to use some elements of particle swarm [19] optimization.

Particle swarm optimization [20] method consists in the following steps:

1) There is a swarm of $S$ particles, and each of them is assigned a coordinate $x_{i} \in \boldsymbol{R}_{\boldsymbol{n}}$ and a velocity $v_{i} \in \boldsymbol{R}_{\boldsymbol{n}}$; $f: \boldsymbol{R}_{\boldsymbol{n}} \rightarrow \mathrm{R}$ is an objective function that needs to be minimized; $p_{i}-$ the best known position of the $i^{\text {th }}$ particle (in the context of solving the given optimization problem); $g$ - the best known state of the entire swarm.

2) For each particle $s_{i} \in S$; $i \in[1 ; S]$ it is needed to:

- generate an initial position using a random vector in the range from $r_{\min }$ to $r_{\max }$ these values are lower and upper boundaries of the search-space, respectively;

- assign to the best known position of particle $p_{i}$ its initial value $x_{i}$; 
- in case $f\left(p_{i}\right)<f(g)$, there is a necessity to update the value from $g$ to $p_{i}$;

- generate velocity value of the particle $v_{i}$, which belongs to the interval from $-\left(r_{\max }-r_{\min }\right)$ to $\left(r_{\max }\right.$ $-r_{\text {min }}$ ).

3) It is required to repeat the following sequencing for each $i^{\text {th }}$ particle until the predetermined stopping criterion is fulfilled:

- generate random vectors $r_{p}$ and $r_{g}$, which have a range of admissible values in the interval between 0 and 1 ;

- update the velocity value of the particle, as in (7):

$v_{i}=w \cdot v_{i}+\varphi_{p} \cdot r_{p} \times\left(p_{i}-x_{i}\right)+\varphi_{g} \cdot r_{g} \times\left(g-x_{i}\right)$

where $\times$ is a vector product operation, $w ; \varphi_{p} ; \varphi_{g}$

- are the parameters specified by user;

- change the particle position according to (8):

$$
x_{i}=x_{i}+v_{i}
$$

- compare the values of $f\left(x_{i}\right)$ and $f\left(p_{i}\right)$; if the first value is less than the second one, it is needful to update the best known position of the particle from $p_{i}$ to $x_{i}$ and then in case $f\left(p_{i}\right)<$ $f(g)$; it is necessary to change the value of the parameter $g$ to the value of the parameter $p_{i}$;

4) As a result of the operations above, the parameter $g$ will contain the best solution.

For pattern recognition quality improvement the authors of the paper developed a clustering method which combines some elements from particle swarm optimization (numerical optimization method) and from $k$-means algorithm (cluster analysis method). From each method such operations were selected that do not require random parameter settings and do not take into account user's subjective opinion (user has just an observing role). In this clustering method all the parameter calculation will happen automatically, and the user no longer needs to generate manually any input values.

It is necessary to normalize the source image before using the developed algorithm. Normalization allows making an image insensitive to the light changes, ridding it of unnecessary noise.

It is achieved by dividing the RGB vector components of each pixel by the length of this vector, as in (9):

$$
\left(r^{\prime}, g^{\prime}, b^{\prime}\right)=\left(\frac{r}{\sqrt{r^{2}+g^{2}+b^{2}}}, \frac{g}{\sqrt{r^{2}+g^{2}+b^{2}}}, \frac{b}{\sqrt{r^{2}+g^{2}+b^{2}}}\right)
$$

where $r, g, b$ are the initial values of pixel's RGB vector; $r$ ', $g^{\prime}, b$ ' are the normalized values of pixel's RGB vector.

The algorithm of the developed clustering method consists in the following procedures:

1) Rounding $W^{\prime}$ pixels horizontally and $H^{\prime}$ pixels vertically to the nearest values of $W$ and $H$, respectively, which are multiple of 10 .
2) Sequential selection of 10 by 10 regions (clusters) in the image and search for a pixel with a maximum average intensity value in each region - these pixels will be centers of mass $c_{j} ; j \in[1 ; W \cdot H / 100]$ (in case there are more than one pixel with a maximum average intensity value in the region, it is possible to choose any of them). The formula of pixel's average intensity calculation is represented in (10):

$$
I_{a v}=\frac{\left(r^{\prime}+g^{\prime}+b^{\prime}\right)}{3}
$$

where $r^{\prime}, g^{\prime}, b^{\prime}$ are the normalized values of pixel's RGB vector.

3) Comparison of the rounded average intensity values for elements with maximum average intensity values from neighboring regions. It was found empirically by authors that the most effective rounding is to two decimal places. If the rounded average intensity values are equal to each other, two neighboring clusters are combined into one. In the new cluster the centroid is the pixel with a maximum average intensity value. It is necessary to repeat this step until there are $M$ clusters $c_{j} ; j \in[1 ; M]$ with the pairwise distinct rounded average intensity values of the centers of mass.

4) Calculation of two parameters for each pixel $x_{i} ; i \in$ $[1 ; W \cdot H]$ relative to each centroid: distance function $d$ and so-called color function $f$. The color function is represented in (11):

$$
f\left(x_{i}, c_{j}\right)=\sqrt{\left(r_{x i}^{\prime}-r_{c j}^{\prime}\right)^{2}+\left(g_{x i}^{\prime}-g_{c j}^{\prime}\right)^{2}+\left(b_{x i}^{\prime}-b_{c j}^{\prime}\right)^{2}}
$$

where $r_{x i}^{\prime} ; g_{x i}^{\prime} ; b_{x i}^{\prime}$ are the normalized [19] values of RGB vector of the pixel $x_{i} ; c_{j}$ is the centroid of the cluster $C_{j} ; r_{c j}^{\prime} ; g_{c j}^{\prime} ; b_{c j}^{\prime}$ are the normalized values of RGB vector of the centroid $c_{j}$.

5) Then for the pixel $x_{i}$ it is necessary to find a centroid $c_{a}, a \in[1 ; M]$ relative to which the square root of distance function value will be minimum and a centroid $c_{b} ; b \in[1 ; M]$ relative to which the value of color function value will be minimum. After that the following differences need to be calculated, as it is represented in (12) and (13):

$$
\begin{aligned}
& d_{\text {diff }}=\left|d\left(x_{i}, c_{a}\right)-d\left(x_{i}, c_{b}\right)\right| \\
& f_{\text {diff }}=\left|f\left(x_{i}, c_{a}\right)-f\left(x_{i}, c_{b}\right)\right|
\end{aligned}
$$

6) The function, whose difference was less, is chosen as a priority function (in case $d_{\text {diff }}$ equals $f_{\text {diff, the }}$ distance function obtains a priority, because pixels that are closer to each other more likely belong to the same object than the ones that have similar colors). The allocation of pixels to clusters is realized according to the priority function, i.e. the pixel $x_{i}$ will be assigned to a cluster, if the priority function value 
between this pixel and this cluster's centroid is minimal.

7) Ridding the image of noise:

For this purpose the authors chose non-local means method. It is illustrated in (14):

$$
u(p)=\frac{1}{C(p)} \int_{\Omega} v(q), f(p, q) d q
$$

where $u(p)$ is the filtered intensity value of pixel color component at point $p, v(q)$ is the unfiltered intensity value of pixel color component at point $q$; $f(p ; q)$ - weighting function, $C(p)$ - normalizing factor.

As the weighting function Gaussian function is used, it is shown in (15):

$$
f(p, q)=e^{\frac{-|B(q)-B(p)|^{2}}{h^{2}}}
$$

where $h$ is the filter parameter (in general, for RGB color images $h=3), B(p)$ is the local average intensity value of color components of pixels around the point $p, B(q)$ is the local average intensity value of color components of pixels around the point $q$. Normalizing factor $C(p)$ is calculated, as in (16):

$$
C(p)=\int_{\Omega} f(p, q) d q
$$

The developed clustering method uses the following elements borrowed from the particle swarm optimization method: particle group motion (pixel-by-pixel passage through the image), search for the best solution for the entire swarm (search for pixel with a maximum average intensity value in a certain region).

At the same time the main differences from the original algorithm are the next points: each particle has a fixed velocity value which excludes the necessity of its manual recalculating by user, initial particle parameters are not specified randomly.

As for the $k$-means cluster analysis method [21], its next aspects were improved: the need of cluster amount predetermining by the user was eliminated, apart from distance function minimization an operation of color function minimization was added, which gave an opportunity to increase the probability that pixels will be assigned to clusters correctly.

\section{EXPERIMENTS}

To check the effectiveness of the clustering method based on particle swarm optimization 860 test images of cars and 860 images of road signs were picked and normalized [22].

For this purpose the mixture of manual photos, images provided by Stanford University laboratory and images from Russian Traffic Sign Dataset (both datasets are publicly available) was used. Authors have analyzed existing pictures with some samples with ideal results of clustering and figure recognition.

Fig. 1 and Fig. 2 outline examples from this set of test images.

The normalization [23] results of these source images are showed in Fig. 3 and Fig. 4.

Fig. 5 and Fig. 6 depict the work results of the clustering method based on particle swarm optimization.

One of the shortcomings of the developed method is that optimal rounding of the average intensity value for cluster combining empirically established by the authors is not universal.

It may provoke two opposite situations:

1) there are extra clusters in the output image, especially in the places of glare;

2) in the output image several different objects are merged into one cluster.

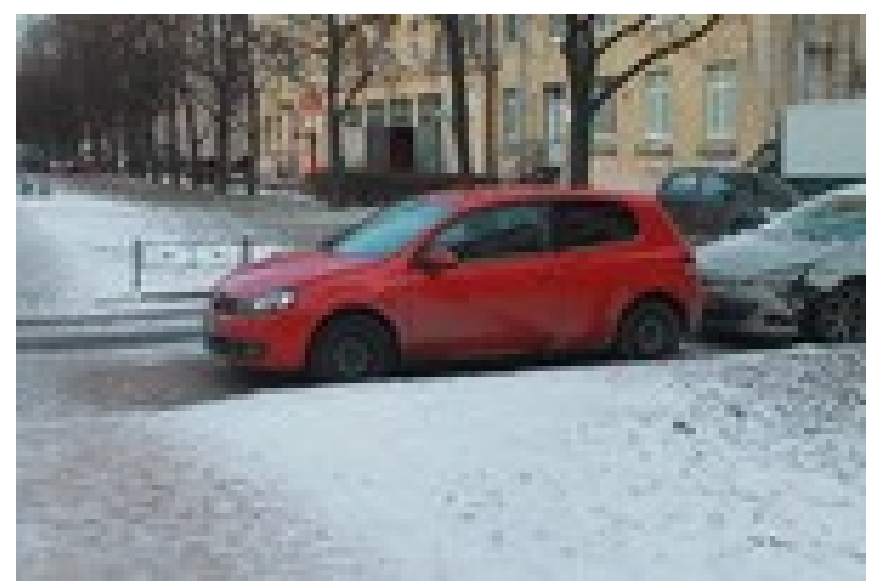

Fig. 1. Source image of a car

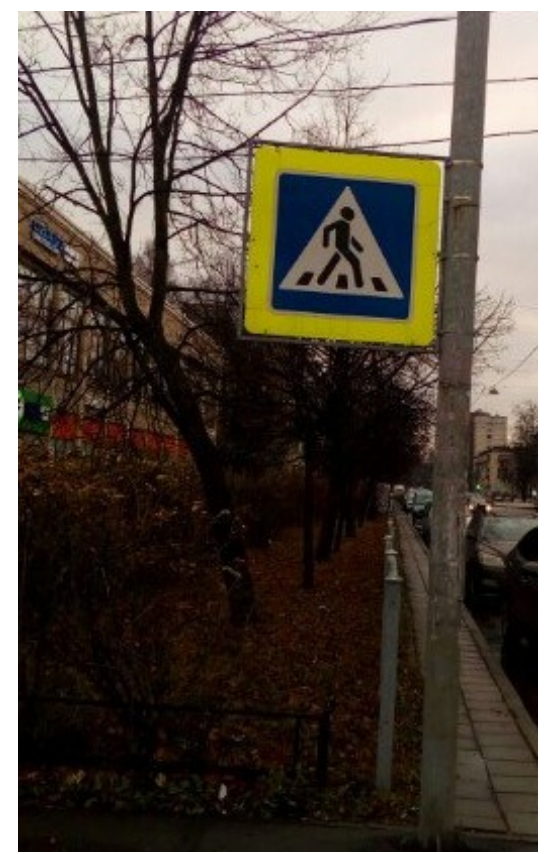

Fig 2. Source image of a road sign 


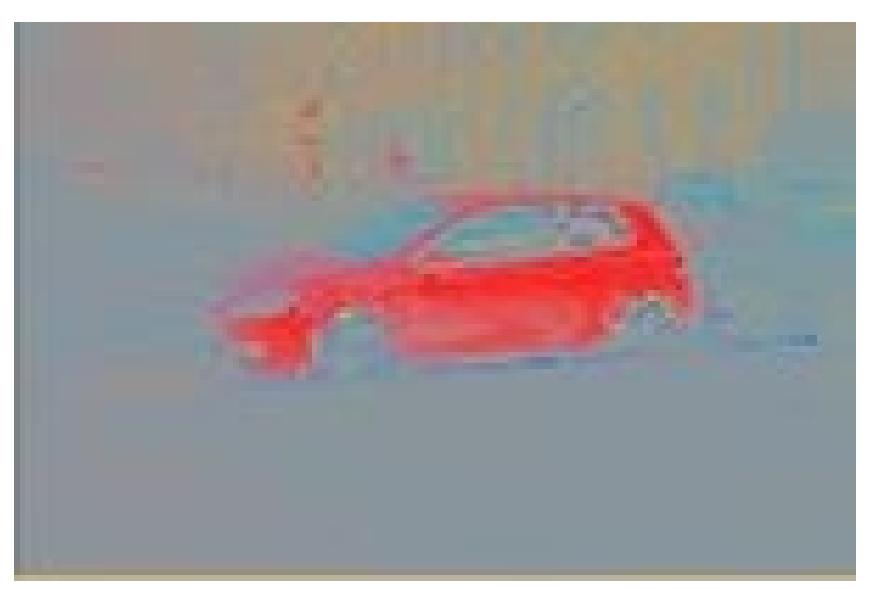

Fig. 3. Normalized image of the car

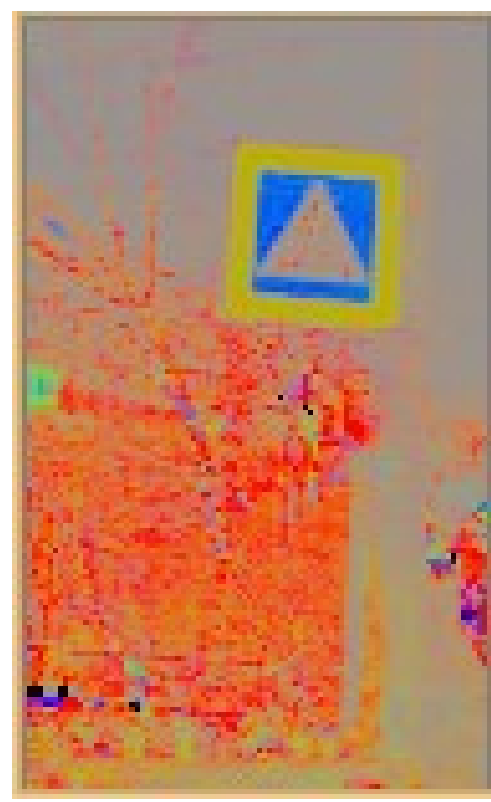

Fig. 4. Normalized image of the road sign

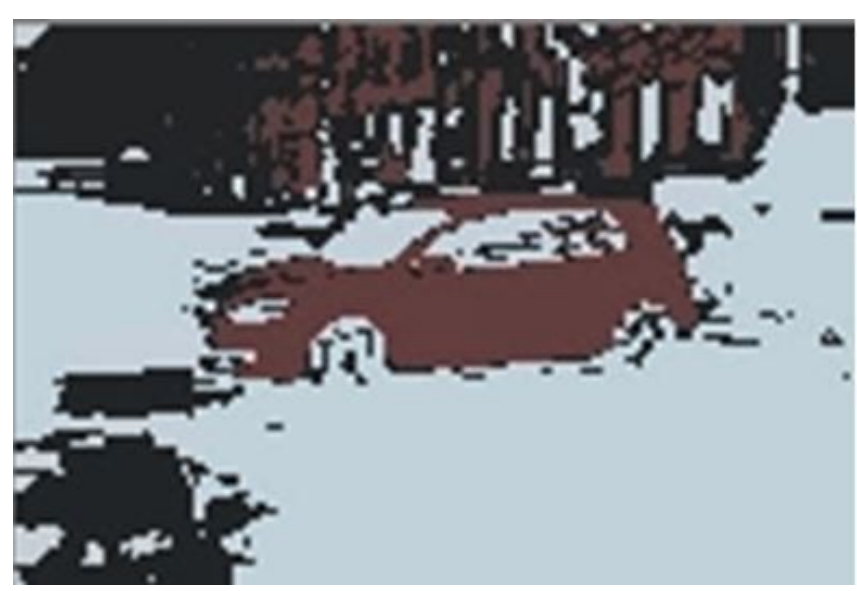

Fig. 5. Clustered image of the car (PSO clustering method)

The examples of work of the clustering method based on the PSO with reduced number of decimal places ( 1 decimal place) in the rounded average intensity value are represented in Fig. 7 and Fig. 8. All the objects were combined into one.
Thus, the image in Fig. 7 seems to be one-colored - the proposed approach can find only one color, so, it seems impossible to recognize any figure on the picture.

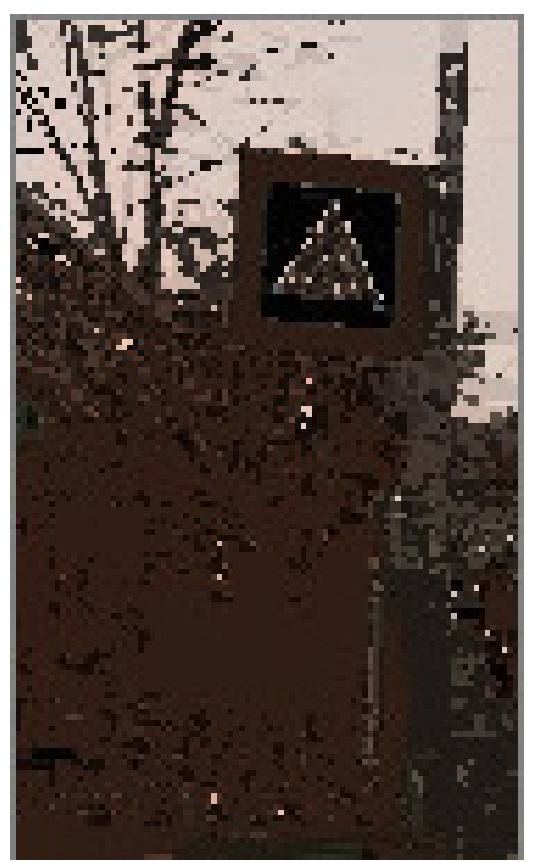

Fig. 6. Clustered image of the road sign (PSO clustering method)

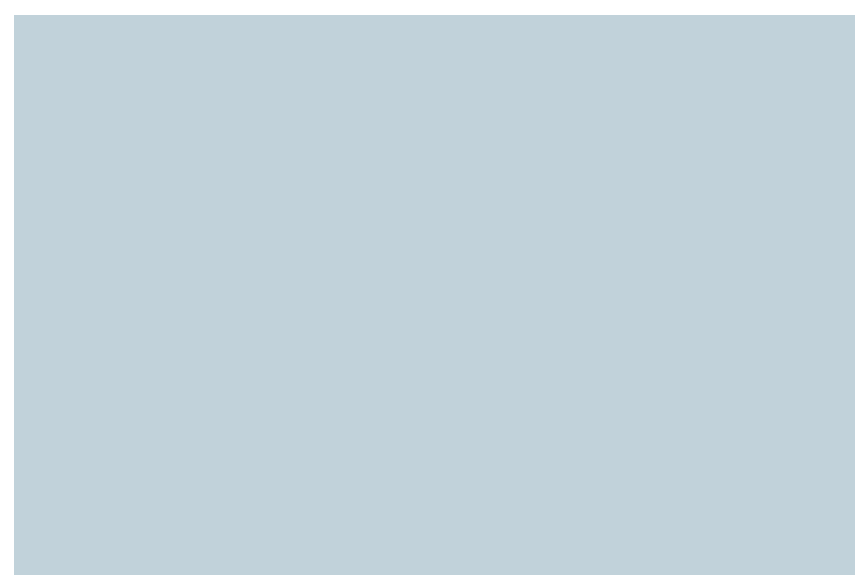

Fig. 7. Example of clustering quality worsening because of reduced number of decimal places in the rounded average intensity value (car image)

The examples of work of the clustering method based on the PSO with increased number of decimal places ( 3 decimal places) in the rounded average intensity value are represented in Fig. 9 and Fig. 10. In the given image there are extra detected regions: piece of land, parts of sky, building.

Also, this method depends on the input image size: the more the number of the analyzed pixels, the greater is risk that more clusters will be detected, and due to this the time of work will be enlarged. This causes the necessity of proportional reducing the source image size or increasing the initial cluster size. 


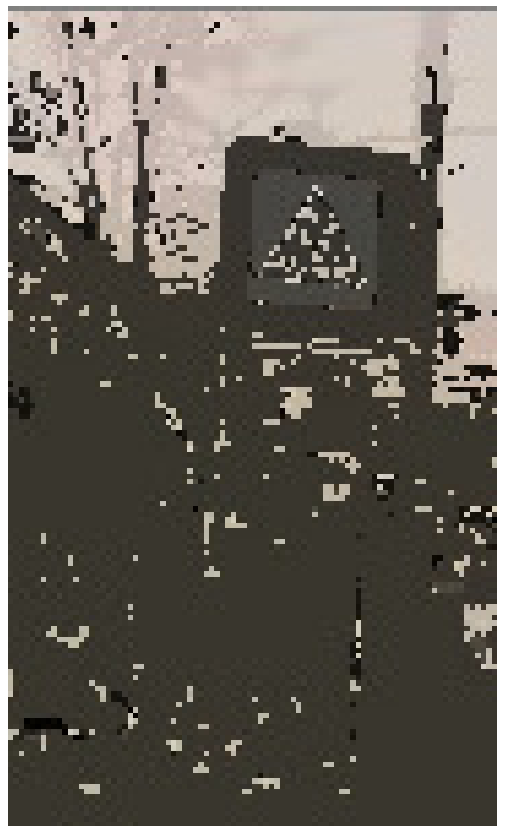

Fig. 8. Example of clustering quality worsening because of reduced number of decimal places in the rounded average intensity value (road sign image)

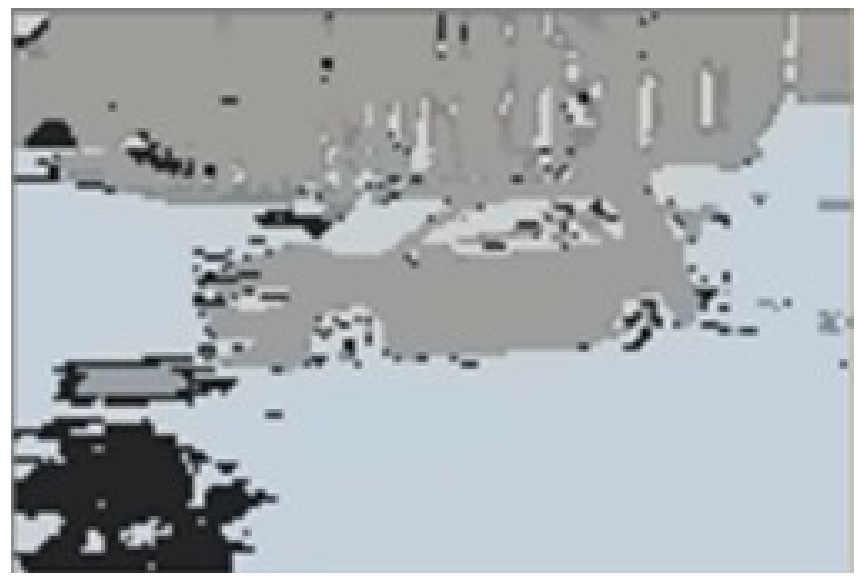

Fig. 9. Example of clustering quality worsening because of increased number

of decimal places in the rounded average intensity value (car image)

The example of work of the clustering method based on the PSO with increased number of resultant cluster quantity due to absence of preliminary image size reduction is represented in Fig. 6 . The size of the source image of the road sign is $500 \times 250$. It is notable that in the resultant image many extra clusters were detected that during further analysis can provoke different issues.

To perform clustering, the authors reduced proportionally the image size. Currently the size of reduced image needs to be established empirically. Particularly, for the images represented in this paper the maximum size of 150 pixels wide and tall was chosen.

Thus, the developed clustering method currently is suitable for small images, which are not overloaded with details and excessive amount of glares.

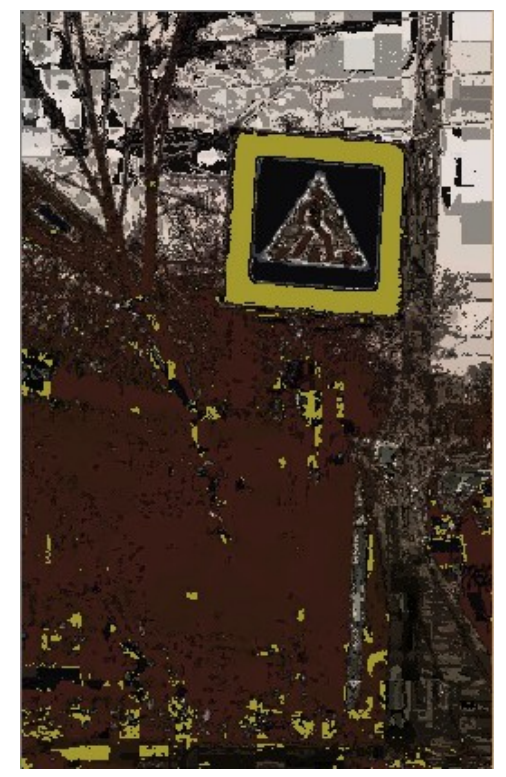

Fig. 10. Example of clustering quality worsening because of increased number of decimal places in the rounded average intensity value (road sign image)

To improve the effectiveness of this method, it is planned to disclose dependencies between image features (pixel quantity, histogram of gradients) and such aspects as: number of decimal places in rounded average intensity value, initial cluster size.

To perform comparison, apart from the clustering method based on the PSO, the test images were clustered by the fuzzy $C$-means and the $k$-means (as an input value, the number of clusters obtained in the PSO clustering method was used; maximum iteration quantity -3 ). The average working time of mentioned clustering methods are represented in Table 1.

TABLE I. COMPARISON TABLE OF TIME OF WORK FOR PSO, FUZZY C-MEANS AND K-MEANS CLUSTERING METHODS

\begin{tabular}{|c|c|c|}
\hline PSO & Fuzzy $C$-means & $k$-means \\
\hline 1.69 & 0.0013 & 35.98 \\
\hline
\end{tabular}

Fig. 13 and Fig. 14 represent the worked results of $k$ means clustering method. Fig. 11 and Fig. 12 show the working results of the fuzzy $C$-means clustering method.

With $k$-means and fuzzy $\mathrm{C}$-means clustering methods it became possible to separate objects from background. However, the algorithm proposed by the authors marks details more legibly.

To illuminate influence of the represented clustering methods on the pattern recognition quality the authors implemented Haar [23] cascade classifier on the given example to detect the car. It is possible to see the results in Fig. 15-20.

PSO and $k$-means clustering methods contributed to obtaining correct results: region with the car was entirely detected. Fuzzy $C$-means algorithm despite its high speed does not guarantee accurate recognition. 


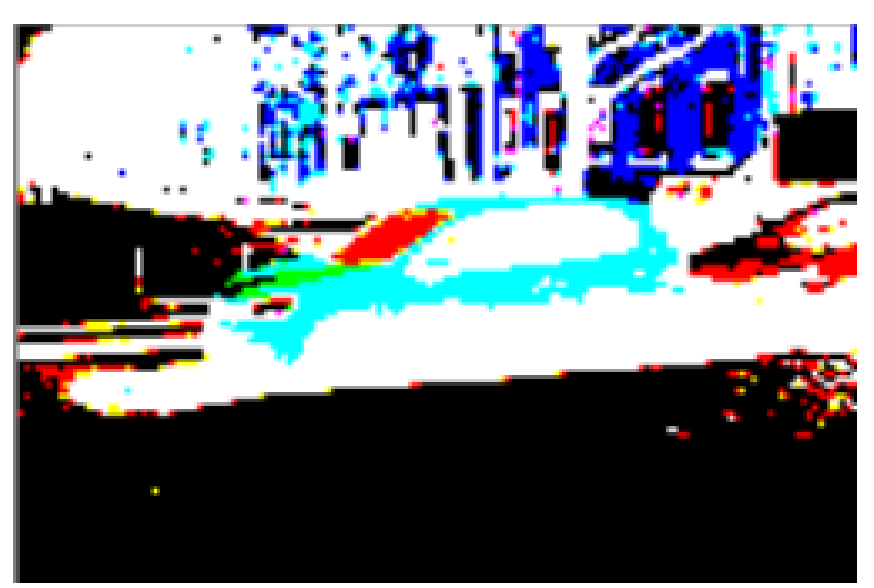

Fig. 11. Clustered image of the car by fuzzy $C$-means clustering method (car image)

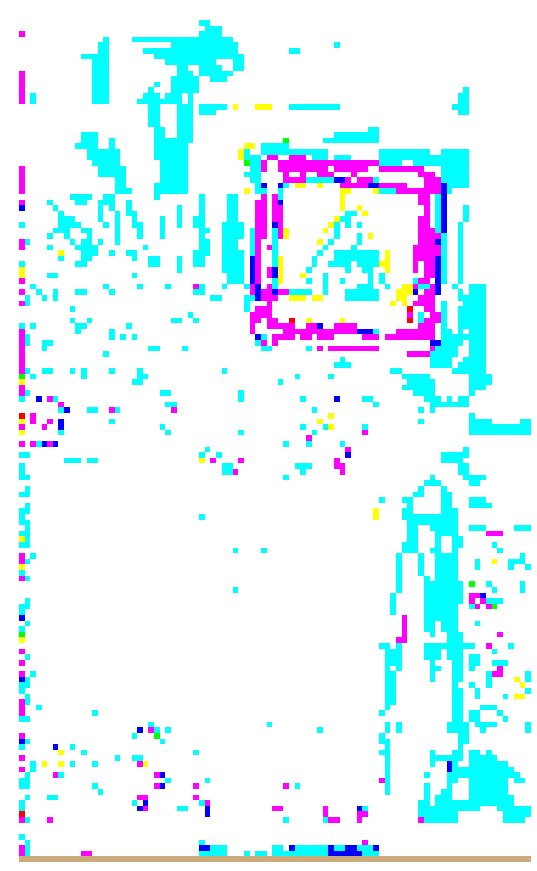

Fig. 12. Clustered image of the car

by fuzzy $C$-means clustering method (road sign image)

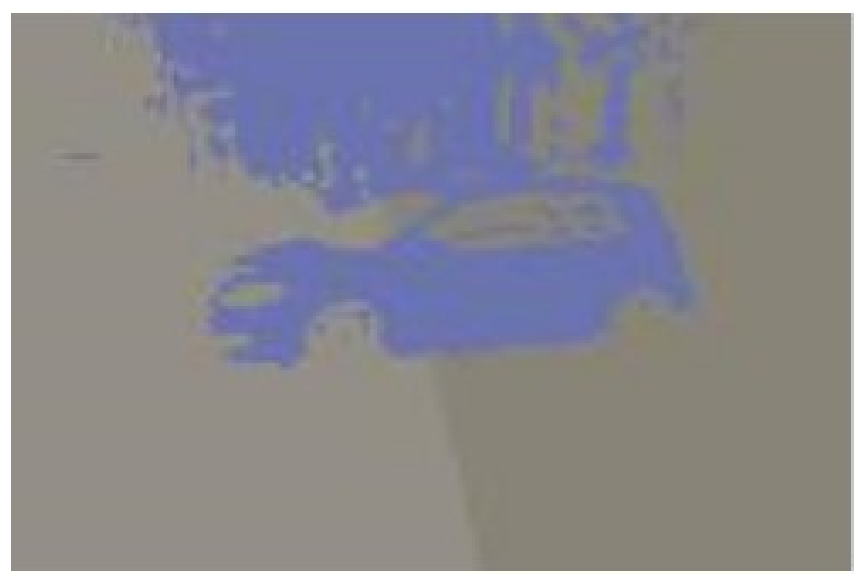

Fig. 13. Clustered image of the car by $k$-means clustering method (car image)

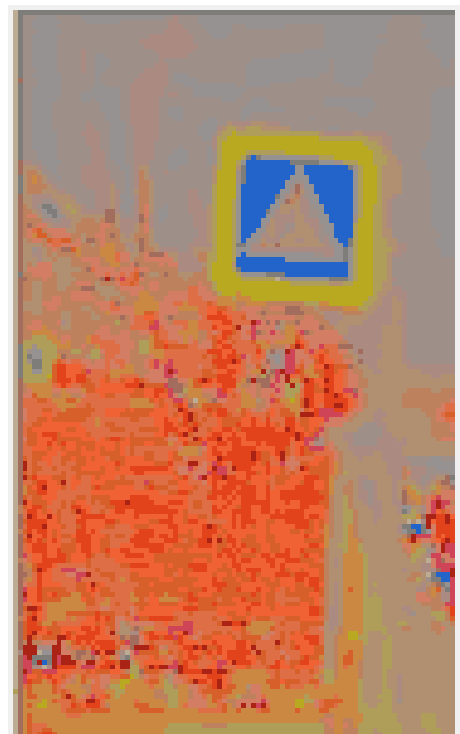

Fig. 14. Clustered image of the car by $k$-means clustering method (road sign image)

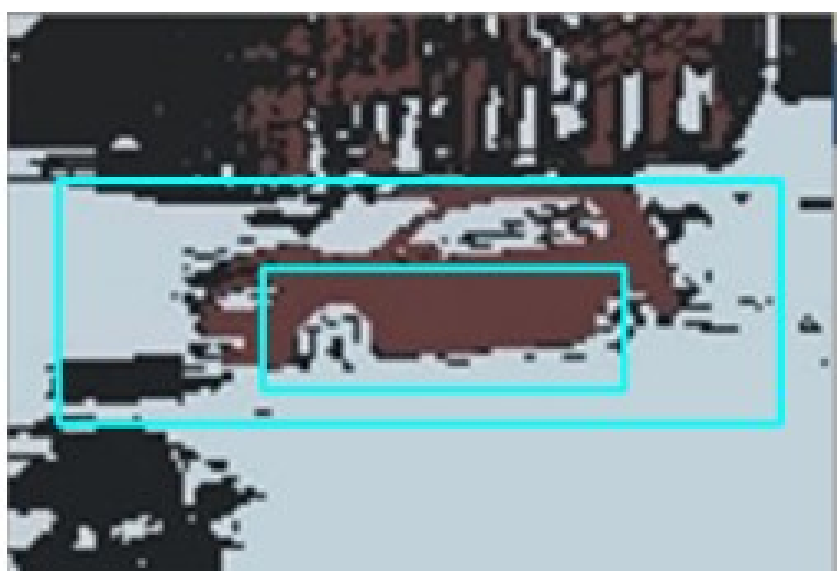

Fig. 15. The result of classifying (PSO clustered image of car)

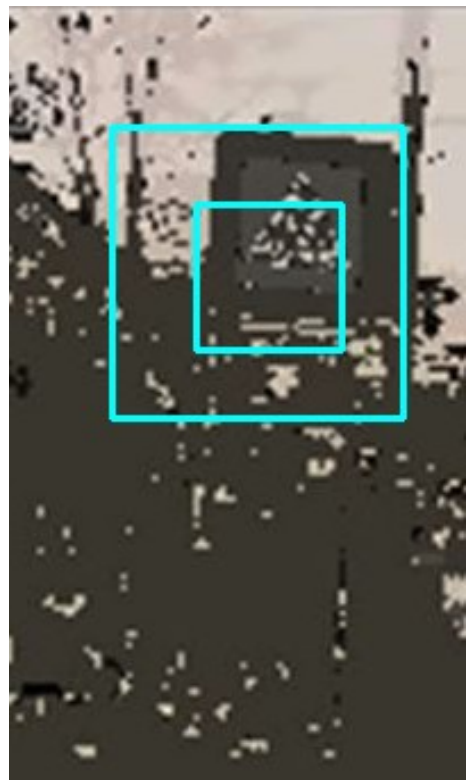

Fig. 16. The result of classifying (PSO clustered image of road sign) 


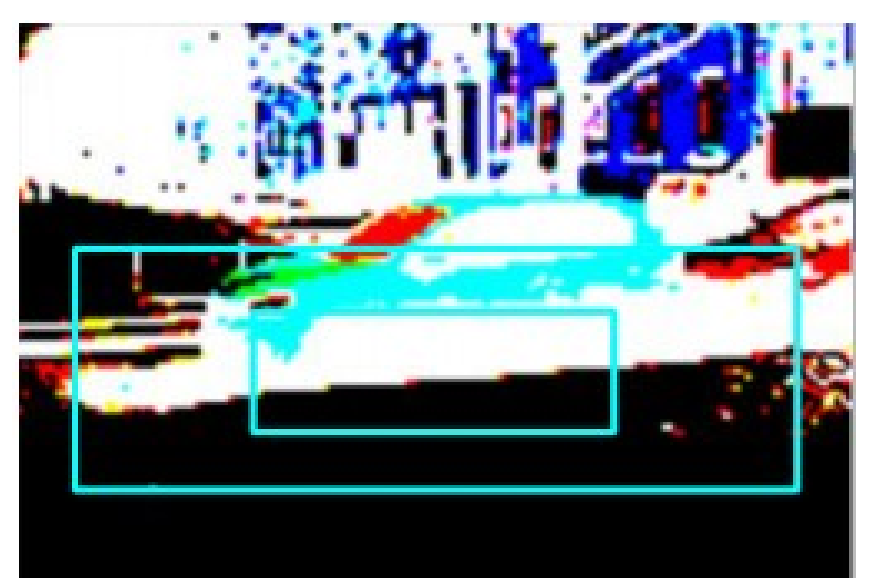

Fig. 17. The result of classifying (fuzzy $C$-means clustered image of car)

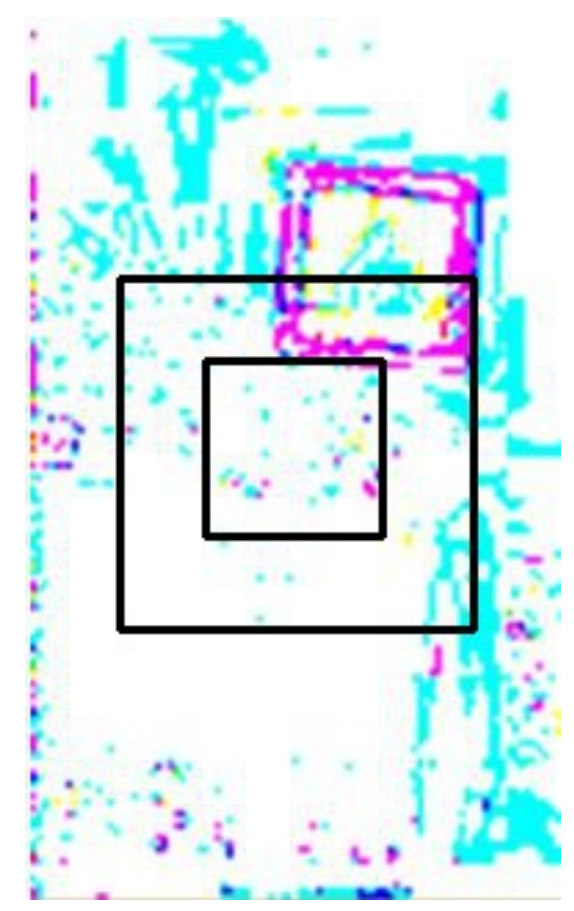

Fig. 18. The result of classifying

(fuzzy $C$-means clustered image of road sign)

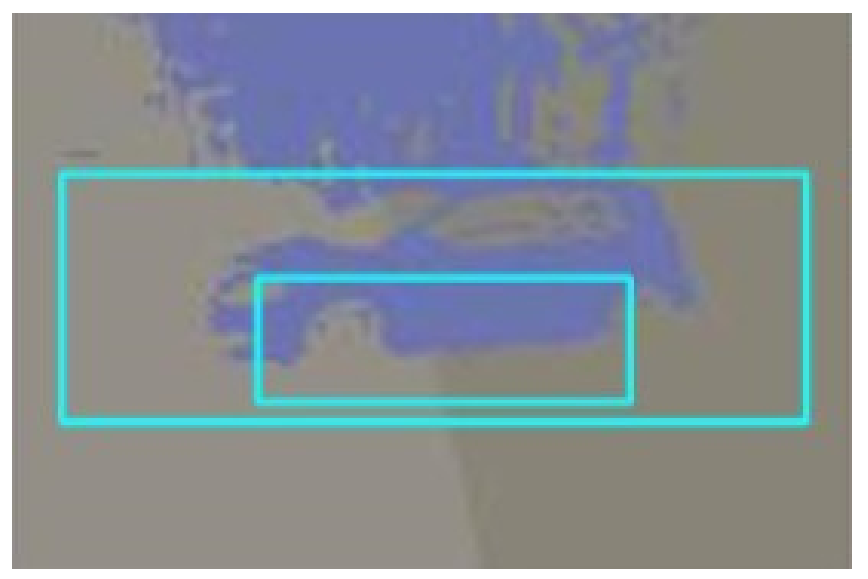

Fig. 19. The result of classifying ( $k$-means clustered image of car)

Comparing PSO and k-means, the second one is slower because of using several iterations.
Reducing iteration number can have a negative impact on clustering quality. PSO determines clusters during the first two steps without the risk of worsening results.

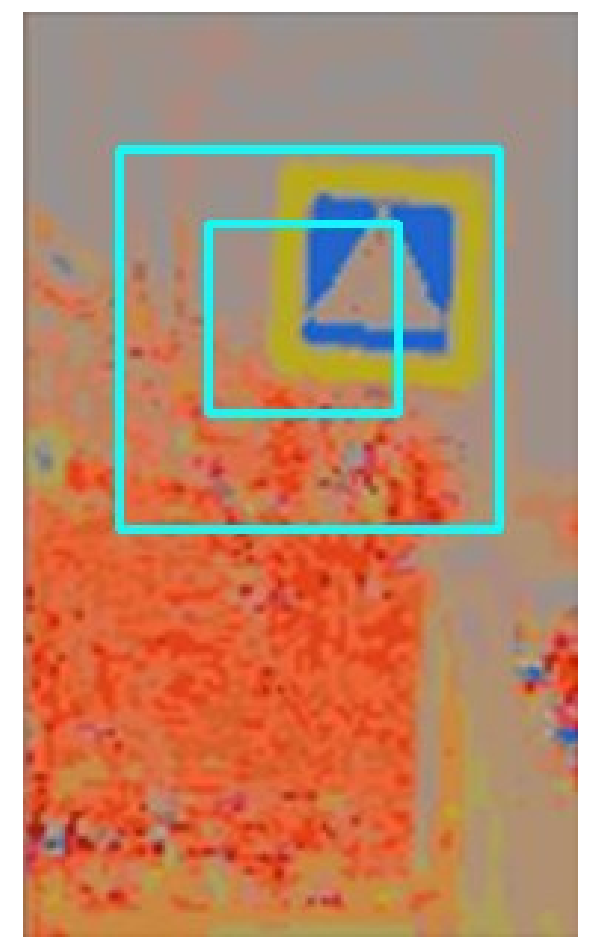

Fig. 20. The result of classifying ( $k$-means clustered image of road sign)

\section{V.CONCLUSION}

The paper proposed and investigated the clustering method based on particle swarm optimization. The developed method was tested for a car vision system and its results and contribution to the pattern recognition quality improvement were evaluated.

The experiments demonstrated that the suggested clustering method is faster than the $k$-means, but it cedes in speed to the fuzzy $C$-means. However, it provides more accurate results. The advantages of the proposed method are as follows:

(1) use of particle swarm optimization helped to eliminate the necessity for user of cluster amount predetermining - one of the main reasons of incorrect clustering in the majority of cluster analysis methods;

(2) the authors liquidated the need for user of calculating and specifying threshold parameters; this need often led to a situation when one pixel could be assigned to several clusters at the same time which contradicts with the clustering task;

(3) there are rules that regulate grouping of objects, which possess the features of the different clusters; thanks to this the uncertainty in correlation of objects and clusters is minimized and, as a result, the pattern recognition error probability is reduced; 
(4) clustering parameters are predetermined automatically and do not require user's intervention.

However, currently, this method has the following disadvantages: absence of clear dependencies between way of rounding the average intensity values and image features; absence of clear dependencies between size of initial cluster and image features.

In further research it is planned to liquidate the mentioned drawbacks of the developed clustering method, propose the ways of its improvement and to finalize the embedded vision prototype. Implementation of the proposed approach may be used for different cyber-physical systems. One of the most interesting sphere is autonomous cars.

Further research will be also aimed on modification of the proposed method and development of software for video streaming analysis for real-time systems. There are such systems for different physical models of autonomous vehicles [24]. Some of them are particular, it is necessary to rebuild algorithm for different conditions of environment, some of them work only with certain functions, required for correct movement of the cars [25-27].

In this case, authors will prepare the method not only for image recognition in usual cases, but also for fully automatic recognition in case of violations. Current results seem to be useful as the main approach for machine vision organization, but this approach should be improved with usage of different existing machine learning methods.

\section{ACKNOWLEDGMENT}

This research is being supported by grants of Russian Foundation for Basic Research (projects No. 16-29-09482 and 18-07-01488), by the budget (project No. AAAA-A16116033110102-5), and by Government of the Russian Federation, Grant 08-08.

\section{REFERENCES}

[1] D. A. Forsyth and J. Ponce. Computer Vision: A Modern Approach. Saddle, River, Nj, U, A: Prentice Hall, 2003.

[2] A. Kochan, "Machine vision guides the automotive industry," Sensor Review. J., vol.22, 2002, pp. 119-124.

[3] D. Vernon. Vernon. Machine Vision in the Electronics and PCB Inspec-tion Industry. The Current Position and Future Directions Maynooth College Ireland, 2004.

[4] S.V.Kozlov, E.Yu.Neretin and V.V. Kukolkina, "Machine vision applica-tion in digital dermoscopy for suspected melanoma of the skin," Saratov Journal of Medical Scientific Research. J., vol.10, 2014, pp. 281-285.

[5] B. Ranft, C. Stiller, "The role of machine vision for intelligent vehicles," IEEE Transactions on Intelligent Vehicles, 2016 Mar;1(1):8-19.

[6] R. Baran, A. Glowacz, A.Matiolanski, "The efficient real-and nonreal-time make and model recognition of cars," Multimedia Tools and Applications, 2015, 74(12), pp.4269-88.

[7] J. Janai, F. Güney, A. Behl, A. Geiger, "Computer vision for autonomous vehicles: Problems, datasets and state-of-the-art," arXiv preprint arXiv:1704.05519. 2017.

[8] A. A. Aly, S. B. Deris, N. Zaki. Research review for digital image segmentation techniques. 2011.
[9] Y. Xiang, A. C. S. Chung, J. Ye, "A new active contour method based on elastic interaction," 2005 IEEE Computer Society Conference on Computer Vision and Pattern Recognition (CVPR'05). vol.1, 2005, pp. 452-457.

[10] N. Li, M. Liu, Y. Li, "Image Segmentation Algorithm using Watershed Transform and Level Set Method," 2007 IEEE International Conference on Acoustics, Speech and Signal Processing - ICASSP '07, Honolulu J., 2007.

[11] R. G. Mathieu, R. L. Woodard, "Data integrity and the Internet: implications for management," Internet Research J., vol.3, 1995, pp. 3-7.

[12] H. Ibrahim, "A strategy for semantic integrity checking in distributed databases," Ninth International Conference on Parallel and Distributed Systems. J., 2002, pp. 139-144.

[13] K. P. Udagepola, L. Xiang, A.W. Wijeratne, Y. Xiaozong, "Semantic integrity constraint violations check for spatial database updating," Journal of Applied Mathematics and Computer Sciences J., vol.4, 2007.

[14] M. Woźniak, D. Połap, "Adaptive neuro-heuristic hybrid model for fruit peel defects detection," Neural Networks, vol. 98, 2018, pp. 1633.

[15] K. Li, W. Tao, X. Liu, L. Liu, "Iterative image segmentation with feature driven heuristic four-color labeling," Pattern Recognition, vol. 76, 2018, pp. 69-79.

[16] R. Geetha Ramani, "Macula segmentation and fovea localization employing image processing and heuristic based clustering for automated retinal screening," Computer methods and programs in biomedicine, vol. 160, 2018, pp. 153-163.

[17] G. T. Huang, K. I. Cunningham, P. V. Benos, C. S. Chennubhotla, "Spectral clustering strategies for heterogeneous decease expression data," Pacific Symposium on Biocomputing. Pacific Symposium on Biocomputing, 2013, pp. 212-223.

[18] J. Lu, Q. Zhu, Q. Wu, "A novel data clustering algorithm using heuristic rules based on k-nearest neighbors chain," Engineering Applications of Artificial Intelligence, vol. 72, 2018, pp. 213-227.

[19] A. P. Karpenko, E. Y. Seliverstov, "Overview of the particle swarm methods for the global optimization problem," Science and Education: a scientific edition of the Bauman Moscow State Technical University J., vol.3, 2009, pp. 1-26

[20] J. Wang, and D. Wang, "Particle swarm optimization with a leader and followers," Progress in Natural Science. J., vol.18, 2008, pp.14371443.

[21] J. Qi, Y. Yu, L. Wang, J. Liu, "K*-Means: An Effective and Efficient K-Means Clustering Algorithm," 2016 IEEE International Conferences on Big Data and Cloud Computing (BDCloud), Social Computing and Networking (SocialCom), Sustainable Computing and Communications (SustainCom) (BDCloud-SocialCom-SustainCom), 2016, pp.242-249.

[22] L. Xie, Q. Tian, B. Zhang, 'Feature normalization for part-based image classification," 2013 IEEE International Conference on Image Processing., Melbourne, 2013, pp. 2607-2611.

[23] S. Choudhury, S. P. Chattopadhyay, T. K. Hazra, "Vehicle detection and counting using haar feature-based classifier," 2017 8th Annual Industrial Automation and Electromechanical Engineering Conference (IEMECON), 2017, pp. 106-109.

[24] B.A. LaPenta, The Ducklingbot: a self-driving robot on a Pi Zero (Doctoral dissertation, Massachusetts Institute of Technology).

[25] V. Kastrinaki, M. Zervakis, K. Kalaitzakis, "A survey of video processing techniques for traffic applications," Image and vision computing, 2003, 21(4), pp.359-81.

[26] J.C. McCall, M.M. Trivedi, "Video-based lane estimation and tracking for driver assistance: survey, system, and evaluation," IEEE transactions on intelligent transportation systems, 2006, 7(1), pp.2037.

[27] M.Y. Fu, Y.S. Huang, "A survey of traffic sign recognition," International Conference on Wavelet Analysis and Pattern Recognition (ICWAPR), 2010, IEEE, pp. 119-124. 